\title{
Group Therapy for Japanese University Students with Autism Spectrum Disorder
}

\author{
Yuko Furuhashi \\ Health Care Center, Shizuoka University, Shizuoka, Japan \\ Email: furuhashi.yuko@shizuoka.ac.jp
}

How to cite this paper: Furuhashi, Y. (2017). Group Therapy for Japanese University Students with Autism Spectrum Disorder. Psychology, 8, 771-780.

https://doi.org/10.4236/psych.2017.85049

Received: March 1, 2017

Accepted: March 28, 2017

Published: March 31, 2017

Copyright $\odot 2017$ by author and Scientific Research Publishing Inc. This work is licensed under the Creative Commons Attribution International License (CC BY 4.0).

http://creativecommons.org/licenses/by/4.0/

\begin{abstract}
Autism spectrum disorder (ASD) is characterized by social impairment and circumscribed and repetitive behavior and interests. This study aimed to examine the effectiveness of group therapy, which was designed to enhance university-related behavior, in Japanese university students with high-functioning ASD, based on their specific social, communication and emotional needs. The participants included 11 students with ASD aged between 19 and 24 (mean 21.0) years. A single-group, pre-post-intervention design was implemented in the study. The Clinical global Impressions-Severity scale, Beck Depression Inventory, State Trait Anxiety Inventory, and Rosenberg SelfEsteem Inventory Scale were completed before and after group therapy. The results showed significant post intervention improvements in depressive symptom, anxiety, and self-esteem, indicating that the group therapy was effective for students with ASD.
\end{abstract}

\section{Keywords}

Autism Spectrum Disorder, University Students, Group Therapy

\section{Introduction}

Autism spectrum disorder (ASD) is characterized by significant impairment in social interaction, verbal and nonverbal communication deficits, and restricted and repetitive interests and behavior (APA, 1994). These symptoms range in severity from mild to severe and usually persist throughout the lifespan. While many individuals with ASD have intact or elevated intellectual ability, non-social neurocognitive impairment has been observed in domains such as processing speed (Mayes \& Calhoun, 2007), aspects of working memory (Williams et al., 2006), planning (Hughes et al., 1994), and executive functioning (Ozonoff, 1994). This constellation of social and non-social deficits in information processing 
limits adaptation and success in adult life significantly, despite normal intellectual ability, in individuals with high-functioning ASD. Furthermore, previous research findings have shown that adults with high-functioning ASD experience significant disadvantage with respect to employment, academic performance, social relationships, mental health, and quality of life (Howlin et al., 2004), as they are vulnerable to numerous psychiatric and neurological disorders such as depression, epilepsy, sleep disorder, anxiety disorder, and obsessive compulsive disorder. Recent reports suggested that adults with ASD were unable to understand their own mental states, which could lead to difficulties in emotional regulation. In addition, hyper- or hyposensitivity to pain, touch, movement, sound, smell, and light have often been reported in this population (Minshew \& Hobson, 2008). These psychiatric and neurological difficulties often lead to impaired quality of life in adults with ASD (Jennes-Coussens et al., 2006). Therefore, there is a need for the development of effective interventions address core cognitive deficits in adults with ASD.

Although antidepressants are used widely in patients with ASD, few studies have been conducted to provide corresponding efficacy data. In contrast, cognitive behavioral therapy (CBT) has shown promising results in the general population (James et al., 2005; Wood et al., 2009) and is safe and acceptable to patients' families. Many studies have demonstrated that CBT was an effective treatment for anxiety and depression in typically developing children and adolescents (Compton et al., 2004; Klein et al., 2005). Various treatments, including CBT, have been developed to alleviate comorbid symptoms in children with ASD. However, it is difficult to adapt psychological methods designed to treat children with ASD to adult patients, because they either involve parental participation or are too time consuming. Interest in the development of CBT based interventions for patients with ASD who continue to experience distress and functional interference because of persistent symptoms has increased. Although CBT has become a widely practiced and empirically supported psychotherapeutic approach, its use in the treatment of ASD has been recommended only in the past decade. In addition, most of the literature regarding ASD focuses on children and adolescents. Unfortunately, comprehensive approaches that address core neurocognitive and socio-cognitive impairment in adults with ASD have yet to be developed. Therefore, there is a need for the development of treatment options for adult patients with ASD.

Group CBT provides the additional benefits increases in specific skills and the minimization of dropout. Moreover, group settings facilitate social interaction and sharing experiences with others, which reduces social isolation.

Therefore, the purpose of this study was to develop an effective group intervention involving CBT for young adults with high-functioning ASD.

\section{Materials and Methods}

\subsection{Participants}

The study included 15 participants recruited from Shizuoka University. All par- 
ticipants fulfilled the criterion, which was a diagnosis of autistic disorder, Asperger's syndrome, or pervasive developmental disorder-not otherwise specified, as defined in the Diagnostic and Statistical Manual of Mental Disorders fourth edition (DSM-IV). In addition, none of the participants had current psychosis, bipolar disorder, or substance-use disorder. The participants provided their written informed consent after receiving a full explanation regarding the study's purpose and procedures.

At the beginning of the therapy, participants were aged between 19 and 24 (mean 21.0) and four of them were female.

In addition, all participants were required to engage in simultaneous individual and group psychotherapy.

\subsection{Procedure}

The study was conducted between April 2014 and March 2016. A single-group, pre-post intervention study design was implemented. The group therapy programs consisted of CBT and recreational activities (Table 1). Group therapy sessions were held biweekly for 6 months and facilitated by a clinical psychologist and a psychiatrist.

Each CBT sessions, each session consisted of the following five components: 1) a warming up; 2) an introduction to the session's topic, in which the importance of the topic was discussed and, the components of the skills were analyzed, discussed, and visualized; 3) a short break, 4 . behavioral practice and feedback, in which skills were practiced using role-play or real life exercises; and 5) a brief evaluation of the session content.

The Clinical global Impressions-Severity (CGI-S), Beck Depression Inventory (BDI), State Trait Anxiety Inventory (STAI), and Rosenberg Self-Esteem Inventory Scale (RSES) were completed upon enrolment and at 24-week follow up.

\subsection{Assessment Measures}

The Clinical Global Impression (CGI) scale measures symptom severity, treatment response, and treatment efficacy in studies involving patients with mental

Table 1. Group therapy programme.

\begin{tabular}{cc}
\hline Session 1 & Session 7 \\
Psycho education on ASD & Game \\
Session 2 & Session 8 \\
Game & Anxiety \\
Session 3 & Session 9 \\
Relaxation & Sports \\
Session 4 & Session 10 \\
Sports & Anger \\
Session 5 & Session 11 \\
Comfort & Coping skills \\
Session 6 & Session 12 \\
Mood monitoring & Summarize participants' progress \\
\hline
\end{tabular}


disorders. The CGI-S represents a valid and reliable alternative for use in routine clinical practice (Pinna et al., 2015). The CGI-S is a seven item instrument via which clinician's rate the severity of patients' illness at the point of assessment, based on their experience with patients with the same diagnosis. Responses are provided using a three-point scale ranging from 1 (Normal, not at all ill) to 3 (Moderately ill). The BDI is 21-items, self-report inventory that measures characteristic attitudes and symptoms of depression (Beck et al., 1961). The original BDI consists of 21 questions regarding the severity of patient's symptoms during the preceding week. There are four possible responses such as 0 (I do not feel sad), 1 (I feel sad), 2 (I am sad all the time and I can't snap out of it), and 3 (I am so sad or unhappy that I can't stand it). A score between 0 and 3is assigned for each item, and total scores are compared to a key to determine the severity of patients' depression. Higher total scores indicate depressive symptoms of greater severity. The BDI-II has been translated and validated in a number of other languages (e.g., Arabic, Chinese, Dutch, French, German, Japanese, and Italian). State Trait Anxiety Inventory (STAI) includes the State Anxiety Scale (S-Anxiety) and the Trait Anxiety Scale (T-Anxiety). The STAI is considered a reliable and valid quantitative tool via which to assess anxiety (Julian, 2011). The S-Anxiety evaluates current state anxiety, which reflects the way in which respondents feel at the point of assessment via items that measure subjective apprehension, tension, nervousness, worry, and activation/arousal of the autonomic nervous system. The T-Anxiety evaluates relatively stable aspects of "anxiety proneness," including general calmness, confidence, and security. The STAI includes 40 items divided equally between the two subscales. Responses for the S-Anxiety subscale indicate intensity of current feelings "at this moment": 1) not at all, 2) somewhat, 3) moderately so, and 4) very much so. Responses for the T-Anxiety scale assess frequency of feelings "in general": 1) almost never, 2) sometimes, 3) often, and 4) almost always. Item scores are added to obtain subtest total scores. Range of scores for each subtest is $20-80$, the higher score indicating greater anxiety. The RSES is considered a reliable and valid quantitative tool via which to assess self-esteem (Rosenberg, 1962). The 10-item scale measures state selfesteem and ask respondents to reflect on their current feelings. It is a ten-item answered on a four-point scale from 0 (strongly agree) to 3 (strongly disagree). It uses a scale of $0-30$, and a score less than 15 indicate problematic low self-esteem.

\subsection{Data Analysis}

All data were analyzed using SPSS version 18.0 (2009) for Windows. Demographic data were analyzed using means and standard deviations (SDs). Pre- and post-intervention data (Week 0 versus week 24) were analyzed using the nonparametric Wilcoxon's signed-rank test. The Mann-Whitney $\mathrm{U}$ test or $\chi^{2}$ test was used to compare the treatment and the drop-out groups, as appropriate. The criterion for statistical significance was considered to be $p<0.05$. 


\section{Results}

\subsection{Patients Characteristics}

Four (26.7\%) of the initial 15 students who began the therapy dropped out prematurely and did not complete the group program. Therefore, the study ultimately included 11 students for whom the therapy was considered suitable. The students' demographic characteristics are shown in Table 2. There were no significant differences in baseline demographic or clinical characteristics between the treatment group and the drop-out group.

\subsection{Comparisons of Pre- and Post-Intervention Scores}

The significant improvement in the mean BDI score was observed $(p=0.008$, the Wilcoxson's single-rank test). In addition, the significant improvements were observed in the mean S-Anxiety score ( $p=0.012$, the Wilcoxson's singlerank test) and the mean RSES score ( $p=0.013$, the Wilcoxson's single-rank test). On the other hand, the mean CGI-S score ( $p=0.372$, the Wilcoxson's single-rank test) and the mean T-Anxiety score $(p=0.178$, the Wilcoxson's single-rank test) did not significantly change throughout the duration of the study (Table 3).

\section{Discussion}

The result of the study suggested that group therapy involving CBT offer a potentially effective treatment for adults with high-functioning ASD.

Although there is limited empirical support for the use of cognitive behavioral techniques in the treatment of adults with high-functioning ASD, there is a growing body of literature based on case studies and a few small, uncontrolled intervention studies suggesting that CBT can be used to treat anxiety, depression, and impaired social skills effectively in this population.

For example, case studies (Cardaciotto \& Herbert, 2004; Hare, 1997) and

Table 2. Demographics.

\begin{tabular}{ccc}
\hline & complete group & drop-out group \\
\hline $\mathrm{n}$ & 11 & 4 \\
mean age (SD) & $21.0(1.18)$ & $21.5(0.56)$ \\
gender male (\%) & $7(63.6)$ & $2(50)$ \\
mean CGI-S (SD) & $3.3(0.6)$ & $3.0(0)$ \\
men BDI (SD) & $12(2.8)$ & $11(3.6)$ \\
mean T-Anxiety (SD) & $62.0(6.7)$ & $63.3(3.4)$ \\
mean S-anxiety (SD) & $63.2(7.4)$ & $64.3(4.1)$ \\
RSES (SD) & $11.02(7.4)$ & $11.25(1.2)$
\end{tabular}

CGI-S: Clinical global impression-severity; BDI: Beck depression inventory; T-Anxiety: Trait anxiety; SAnxiety: State anxiety; RSES: The Rosenberg self-esteem scale. 
Table 3. Comparisons baseline and endpoint.

\begin{tabular}{ccc}
\hline & baseline (0 W) & endpoint (24 w) \\
\hline mean CGI-S (SD) & $3.3(0.6)$ & $2.9(0.5)$ \\
men BDI (SD) & $12.3(2.8)$ & $8.6(3.1)$ \\
mean T-Anxiety (SD) & $62.9(6.7)$ & $61.3(6.1)$ \\
mean S-anxiety (SD) & $63.2(7.4)$ & $56.5(5.4)$ \\
RSES (SD) & $11.0(1.5)$ & $13.4(1.7)$ \\
\hline
\end{tabular}

studies examining group CBT for individuals with high-functioning ASD (Weiss \& Lunsky, 2010; Eack et al., 2013; Pugliese \& White, 2014) have demonstrated the feasibility and acceptability of group CBT as a treatment method. Despite these positive findings from single-group studies, the relatively small numbers of participants and lack of randomly assigned comparison groups limit the generalizability of the findings, and the observed improvements could be attributable to participant characteristics.

Three randomized controlled trials (RCTs) have explored the use of CBT for mental health problems in adults with high-functioning ASD. For example, McGillivray and Evert (2014) examined the efficacy of group CBT in reducing symptoms of depression, anxiety, and stress in young adults with ASD. According to Hesselmark et al. (2014) and Russell et al. (2013), the results of RCTs showed that group CBT improved social behavior, symptoms of obsessive compulsive disorder, and general ASD symptoms. However, the findings were mixed with respect to self-esteem, distress, and anxiety.

Some studies examining group CBT in adults with high-functioning ASD have demonstrated various benefits following treatment, but others showed that these benefits were absent at follow-up assessment. In addition, grater effectiveness in single-group, relative to randomized studies. However, Tarrier and Wykes (2004) pointed out that studies using poorer methodology in examining the use of CBT to treat psychosis often overestimated the benefits of treatment. Moreover, randomized studies are more reliable relative to non-blinded assessments, which are associated with exaggerated effects.

While single-group studies and RCT CBT differed with respect to various factors, such as intervention durations, numbers of sessions, treatment comparison, and post-intervention and follow-up outcomes, a number of common findings emerged.

For example, CBT found to be particularly effective as an adjunct to medication for patients with ASD. These results are consistent with those of the current study. Further, cognitive rehabilitation represents a potentially effective approach to the remediation of impaired information processing in individuals with high-functioning ASD.

$\mathrm{CBT}$ is a form of psychotherapy that was originally developed to treat depression in the general population. The defining feature of CBT is that the mechanism of action for clinical improvement is a change in cognition, via which dysfunctional cognitive structures are corrected. Furthermore, it provides structure 
and predictability, which makes CBT an attractive treatment option for patients with ASD (Gaus, 2011). However, CBT, programs for individuals with ASD should include psycho-education regarding ASD, related psychiatric symptoms, and social interaction (Gerhardt \& Lainer, 2011). Individuals with ASD could feel like outsiders within groups of typically developing peers, which induces feelings of isolation, ending to anxiety and depressive mood. However, several studies involving children with ASD have demonstrated significant reductions in anxiety symptoms following participation in manualized CBT group therapy interventions (Reaven et al., 2012; Chalfant et al., 2007; Sofronoff et al., 2005). These results are particularly promising given the high rates of comorbidity between ASD and anxiety (Attwood, 2005). In addition, they provide initial support for the feasibility of implementing CBT in group setting for participants with ASD.

In the current study, participants indicated that they considered the group meetings the most helpful component of the therapy. Moreover, in the post intervention questionnaire, all participants reported positive experiences in meeting others with ASD. They also stated that it was helpful to meet other patients with ASD, to see that many of the group members share the same problems, and to learn how others with ASD solve their problems. Therefore, the group setting could have helped to normalize their problems.

While there are some pragmatic issues that require consideration, group modality offers greater benefits, relative to those provided by individual interventions, for various reasons. Despite the diverse symptomatology in ASD, participants experienced similar problems, and group therapy created a forum via which they could offer advice, strategies, and insight to other group members. While individuals with ASD could feel like outsiders within groups of typically developing peers, the group therapy modality provides patients with an opportunity to feel accepted and understood by a group of peers with the same disorder and practice problem-solving skills in a naturalistic but safe and supportive environment. Thus, group therapy could provide understanding, a sense of normality, and a safe space within which to discuss an isolated experience.

It is difficult for adults with high-functioning ASD in terms of adults. Transition into adulthood is often a challenging time for young people with ASD. Social impairment, which is inherent in high-functioning ASD, interferes with establishment of relationships, occupational functioning, and community participation and integration, which are all aspects of social functioning that prominent during the transition to adulthood. The management of these new social demands is difficult for many adults with high-functioning ASD. Recognition of these challenges has contributed to the development of treatments that aim to increase functioning by focusing on selected social skills, which could improve social cognition and functioning in adults with high-functioning ASD. However, few evidence-based interventions for adults with ASD involve social skills or cognition.

Nevertheless, CBT should be considered a component of widespread treatment that combines social interventions and medication. However, service deli- 
very is limited because the number of trained therapists is insufficient and further research is required to examine the functioning elements of CBT.

\section{Conclusion}

The number of adults with high-functioning ASD, seeking help via psychotherapy has increased.

This study sought to address the shortage of treatment options for adults with high-functioning ASD, and the results suggested that group therapy involving CBT could be efficacious in this population.

Future directions for CBT research include robust and well-designed studies examining the effective features of CBT. In addition, future research should focus on component analysis to evaluate the mechanisms via which change occurs, which could ultimately lead to efficient and effective interventions for adults with high-functioning ASD. Furthermore, there is a need for a robust measurement instrument via which to examine relapse, which should be applied consistently across studies. In addition, the use of CBT to reduce relapse should be explored for patients prone to relapse and remission.

While the results indicated that group CBT was effective, further work is required to refine CBT and identify the most effective components and suitable methods of delivery. Moreover, rigorous research designs involving methodologically strict RCT are required to compare of treatments and independent evaluation should be performed to determine treatment efficacy.

\section{Acknowledgements}

This work was supported by JSPS KAKENHI Grant Number JP26381317.

\section{Conflict of Interest}

The authors have no conflicts of interest to declare.

\section{References}

American Psychiatric Association (1994). Diagnostic and Statistical Manual of Mental Disorders (DSM- $I V)$. Washington DC: APA.

Attwood, T (2005). Cognitive Behavioral Therapy for Children and Adults with Asperger's Syndrome. Behavior Change, 21, 147-161. https://doi.org/10.1375/bech.21.3.147.55995

Beck, A. T., Ward, C. H., Mendelson, M., Mock, J., \& Erbaugh, J. (1961). An Inventory for Measuring Depression. Archives of General Psychiatry, 4, 561-571. https://doi.org/10.1001/archpsyc.1961.01710120031004

Cardaciotto, L., \& Herbert, J. D. (2004). Cognitive Behavioe Therapy for Social Anxiety Disorder in the Context of Asperger's Syndrome: A Single-Subject Report. Cognitive and Behavioral Practice, 11, 75-81.

Chalfant, A. M., Rapee, R., \& Carroll, L. (2007). Treating Anxiety Disorders in Children with High Functioning Autism Spectrum Disorders: A Controlled Trial. Journal of Autism and Developmental Disorders, 37, 1842-1857.

https://doi.org/10.1007/s10803-006-0318-4 
Compton, S. N., March, J. S., Brent, D. et al. (2004). Cognitive Behavioral Psychotherapy for Anxiety and Depressive Disorders in Children and Adolescents: An Evidence Based Medicine Review. Journal of the American Academy of Child and Adolescent Psychiatry, 43, 930-959. https://doi.org/10.1097/01.chi.0000127589.57468.bf

Eack, M. S., Greenwald, D. P., Hogarty, S. S. et al. (2013). Cognitive Enhancement Therapy for Adults with Autism Spectrum Disorder: Results of an 18-Month Feasibility Study. Journal of Autism and Developmental Disorders, 43, 2866-2877. https://doi.org/10.1007/s10803-013-1834-7

Gaus, V. L. (2011). Cognitive Behavioural Therapy for Adults with Autism Spectrum Disorder. Advances in Mental Health and Intellectual Disabilities, 5, 15-25. https://doi.org/10.1108/20441281111180628

Gerhardt, P. F., \& Lainer, I. (2011). Addressing the Needs of Adolescents and Adults with Autism: A Crisis on the Horizon. Journal of Contemporary Psychotherapy, 41, 37-45. https://doi.org/10.1007/s10879-010-9160-2

Hare, D. J. (1997). The Use of Cognitive-Behavioral Therapy with People with Asperger Syndrome: A Case Study. Autism, 1, 215-225. https://doi.org/10.1177/1362361397012007

Hesselmark, E., Plenty, S., \& Bejerot, S. (2014). Group Cognitive Behavioral Therapy and Group Recreational Activity for Adults with Autism Spectrum Disorders: A Preliminary Randomized Controlled Trial. Autism, 18, 672-683. https://doi.org/10.1177/1362361313493681

Howlin, P., Goode, S., \& Rutter, M. (2004). Adult Outcome for Children with Autism. Journal of Child and Psychiatry, 45, 212-229. https://doi.org/10.1111/j.1469-7610.2004.00215.x

Hughes, C., Russell, J., \& Robbins, T. W. (1994). Evidence for Executive Dysfunction in Autism. Neuropsychologia, 32, 477-492.

James, A., Soler, A., \& Weatherall, R. (2005). Cognitive Behavioral Therapy for Anxiety Disorders in Children and Adolescents. Cochrane Database of Systematic Reviews, No. 4, Article No: CD004690. https://doi.org/10.1002/14651858.CD004690.pub2

Jennes-Coussens, M., Magill-Evans, J., \& Koning, C. (2006). The Quality of Life of Young Men with Asperger Syndrome: A Brief Report. Autism, 10, 403-414. https://doi.org/10.1177/1362361306064432

Julian, L. J. (2011). Measures of Anxiety: State-Trait Anxiety Inventory (STAI), Beck Anxiety Inventory (BAI), and Hospital Anxiety and Depression Scale-Anxiety (HADS-A). Arthritis Care \& Research, 63, S467-S472. https://doi.org/10.1002/acr.20561

Klein, A., Pauls, R., Schultz, R. et al. (2005). Three Diagnostic Approaches to Asperger Syndrome: Implications for Research. Journal of Autism and Developmental Disorders, 35, 221-234. https://doi.org/10.1007/s10803-004-2001-y

McGillivray, J. A., \& Evert, H. T. (2014). Group Cognitive Behavioral Therapy Program Shows Potential in Reducing Symptoms of Depression and Stress among Young People with ASD. Journal of Autism and Developmental Disorders, 44, 2041-2051.

https://doi.org/10.1007/s10803-014-2087-9

Minshew, N. J., \& Hobson, J. A. (2008). Sensory Sensitivities and Performance on Sensory Perceptual Tasks in High-Functioning Individuals with Autism. Journal Autism Developmental Disorders, 38, 1485-1498. https://doi.org/10.1007/s10803-007-0528-4

Ozonoff, S., Strayer, D. L., McMahon, W. M. et al. (1994). Executive Function Abilities in Autism and Tourette Syndrome: An Information Processing Approach. Journal of Child Psychology and Psychiatry, 35, 1015-1032.

https://doi.org/10.1111/j.1469-7610.1994.tb01807.x

Pinna, F., Deriu, L., Diana, E., Perra, V., Randaccio, R. P., Sanna, L., Tusconi, M., Carpi- 
niello, B., \& Cagliari Recovery Study Group (2015). Clinical Global Impression-Severity Score as a Reliable Measure for Routine Evaluation of Remission in Schizophrenia and Schizoaffective Disorders. Annals of General Psychiatry, 14, 6.

https://doi.org/10.1186/s12991-015-0042-6

Pugliese, C. E., \& White, S. W. (2014). Brief Report: Problem Solving Therapy in College Students with Autism Spectrum Disorders: Feasibility and Preliminary Efficacy. Journal of Autism and Developmental Disorders, 44, 719-729.

https://doi.org/10.1007/s10803-013-1914-8

Reaven, J., Blakeley-Smith, A., Culhane-Shelburne, K. et al. (2012). Group Cognitive Behavior Therapy for Children with High-Functioning Autism Spectrum Disorders and Anxiety: A Randomized Trial. Journal of Child Psychology and Psychiatry, 53, 410-419. https://doi.org/10.1111/j.1469-7610.2011.02486.x

Rosenberg, M. (1962). The Association between Self-Esteem and Anxiety. Journal of Psychiatric Research, 1, 135-152. https://doi.org/10.1016/0022-3956(62)90004-3

Russell, A. J., Jassi, A., Fullana, M. A. et al. (2013). Cognitive Behavior Therapy for Comorbid Obsessive-Compulsive Disorder in High-Functioning Autism Spectrum Disorders: A Randomized Controlled Trail. Depression and Anxiety, 30, 697-708. https://doi.org/10.1002/da.22053

Sofronoff, K., Attwood, T., \& Hinton, S. (2005). A Randomized Controlled Trial of a CBT Intervention for Anxiety in Children with Asperger Syndrome. Journal of Child Psychology and Psychiatry, 46, 1152-1160. https://doi.org/10.1111/j.1469-7610.2005.00411.x

Tarrier, N., \& Wykes, T. (2004). Is There Evidence That Cognitive Behaviour Therapy Is an Effective Treatment for Schizophrenia? A Cautions or Cautionary Tale? Behaviour Research and Therapy, 42, 1377-1401.

Weiss, J. A., \& Lunsky, Y. (2010). Group Cognitive Behaviour Therapy for Adults with Asperger Syndrome and Anxiety or Mood Disorder: A Case Series. Clinical Psychology and Psychiatry, 17, 438-446. https://doi.org/10.1002/cpp.694

Williams, J. H., Waiter, G. D., Gilchrist, A. et al. (2006). Neural Mechanisms of Imitation and "Mirror Neuron" Functioning in Autistic Spectrum Disorder. Neuropsychologia, $44,610-621$.

Wood, J. J., Drahola, A., Sze, K. et al. (2009). Cognitive Behavioral Therapy for Anxiety in Children with Autism Spectrum Disorders: A Randomized Controlled Trail. Journal of Child Psychology and Psychiatry, 50, 224-234.

https://doi.org/10.1111/j.1469-7610.2008.01948.x 
Submit or recommend next manuscript to SCIRP and we will provide best service for you:

Accepting pre-submission inquiries through Email, Facebook, LinkedIn, Twitter, etc. A wide selection of journals (inclusive of 9 subjects, more than 200 journals)

Providing 24-hour high-quality service

User-friendly online submission system

Fair and swift peer-review system

Efficient typesetting and proofreading procedure

Display of the result of downloads and visits, as well as the number of cited articles Maximum dissemination of your research work

Submit your manuscript at: http://papersubmission.scirp.org/

Or contact psych@scirp.org 